\title{
La competencia lectora del alumnado universitario en contexto AICLE
}

\author{
José Manuel Foncubierta \\ Universidad de Huelva
}

Francisco Herrero Machancoses

Fundación Andaluza Beturia para la Investigación en Salud (FABIS)

\author{
M. CARMEn FonseCA-Mora \\ Universidad de Huelva
}

Received: $16^{\text {th }}$ October $2017 /$ Accepted: $20^{\text {th }}$ January 2018

ISSN: $1697-7467$

\begin{abstract}
RESUMEN: La competencia lectora en lengua extranjera del alumnado en contexto AICLE (Aprendizaje Integrado de Contenidos y Lenguas Extranjeras) constituye una de las destrezas cruciales para aprehender el contenido. El objetivo fundamental de este artículo es el análisis de la fluidez lectora silenciosa para reconocer el comportamiento de la competencia lectora del alumnado universitario. Para ello, se han recogido datos mediante pruebas de segmentación de palabras en lengua materna y en lengua extranjera, así como datos de una prueba que diagnostica su nivel de comprensión lectora en lengua extranjera. Los principales resultados muestran el bajo nivel de competencia lectora del alumnado y plantea la utilidad de realizar mediciones de lectura silenciosa para diagnosticar sus dificultades lectoras.

Palabras clave: competencia lectora, lectura silenciosa, AICLE, fluidez lectora, lengua extranjera.
\end{abstract}

\section{University Students' Reading Competence in CLIL Context}

\begin{abstract}
Foreign-language students' reading competence in CLIL (Content and Language Integrated Learning) contexts constitutes one of the critical skills to grasp content. The main objective of this article is to analyze silent reading fluency to observe university students' reading competence. Data has been collected using a word segmentation test in first and foreign language, as well as a test that diagnosed their foreign language reading comprehension level. The main results show students' low level of reading competence and poses the usefulness of measuring students' silent reading to diagnose their reading difficulties.
\end{abstract}

Keywords: reading competence, silent reading, CLIL, reading fluency, foreign language.

\section{INTRODUCCIÓN}

En un mundo cada vez más global y digitalizado, la adquisición de una buena competencia lectora plurilingüe permite al alumno universitario el acceso a un sistema laboral cada vez más dinámico y deslocalizado, así como un mejor desempeño como agente social en las diferentes sociedades receptoras. Leer con fluidez y comprender lo que se lee en una segunda lengua (L2) es una competencia transversal compleja, de naturaleza interlingüística 
y multidimensional, que requiere del dominio de diferentes destrezas. De acuerdo con el Consejo de Europa, la competencia lectora se define como "la habilidad general de comprender, usar y reflexionar sobre las distintas formas del lenguaje escrito con el objeto de alcanzar un desarrollo personal y social satisfactorio" (Eurydice, 2011: 7).

En lengua materna (L1), hay un consenso general sobre el valor correlacional de la fluidez lectora oral y la comprensión de textos escritos en la población infantil y adolescente, siendo así un indicador general de competencia lectora (Fuchs et al., 2001). Rasinski (2014) afirma que a mayor velocidad lectora corresponde una mayor capacidad de comprensión. En una segunda lengua, sin embargo, el proceso lector es aún más complejo que en L1 y la investigación sobre la fluidez lectora oral no ha arrojado aún resultados concluyentes (Lems, 2003; Jeon, 2012), a la vez que los estudios sobre el comportamiento de la fluidez en la lectura silenciosa (FLS) son aún escasos, especialmente en el nivel universitario.

El objetivo central de este estudio es examinar si la FLS podría ser un indicador de progresión y de desarrollo de la competencia lectora de los estudiantes jóvenes universitarios en un contexto AICLE, donde confluyen diferentes niveles de competencia comunicativa en inglés como segunda lengua. De los dos modos de lectura, la oral y la silenciosa, hemos decidido investigar esta última por ser el tipo de lectura natural en la vida adulta, "el modo de lectura de referencia en las sociedades basadas en el conocimiento" (Share, 2008: 594). Por ser, en definitiva, el modo de lectura competente (van den Boer, van Bergen, \& de Jong, 2014).

\section{Marco teórico y ReVisión de la literatura}

\subsection{La fluidez en el proceso lector}

De acuerdo con el grupo de estudio RAND (2002: 11), "la comprensión lectora es el proceso de extraer y construir simultáneamente el significado a través de la interacción y la implicación con la lengua escrita". En lengua materna (L1), hay una abundante cantidad de estudios basados en la medición de la fluidez lectora oral para identificar el desarrollo del proceso lector y a los alumnos con dificultades potenciales de aprendizaje, al ser este el modo de lectura con que los niños van adquiriendo la relación entre el código escrito y la lengua oral. En el nivel educativo elemental, cuando los lectores noveles van adquiriendo la relación grafo-fonológica y aprenden a identificar palabras en los textos escritos (decodificación), la fluidez lectora oral, entendida como una cualidad que expresa un porcentaje importante de corrección, velocidad y expresividad en la lectura (Kuhn, Schwanenflugel, Meisinger, Levy, \& Rasinski, 2010; National Reading Panel, 2000; T. Rasinski \& Samuels, 2011), suele correlacionar muy significativamente con la capacidad de comprender el significado de la lengua escrita. Los niños con problemas de decodificación presentan dificultades para comprender lo que leen.

En lo que respecta al estudio de la FLS, para Hiebert, Samuels, \& Rasinski (2012) y Ciuffo et al. (2017), la lectura en silencio supera al desarrollo de la fluidez lectora oral, ya que el techo máximo de desarrollo y eficiencia ocupa toda la vida educativa hasta seguir 
incrementándose, incluso, en el nivel universitario. Ciuffo et al., (2017: 1679) definen la FLS como "la habilidad de leer con concentración, a una velocidad adecuada y con una buena comprensión".

En la actualidad, los sistemas de medición de la fluidez lectora son principalmente orales. Se dispone de marcos normativos con índices de lectura fluida en palabras por minuto, se practican en el contexto de la lengua materna y se emplean fundamentalmente en las escuelas. Después de la enseñanza primaria, apenas hay medición de la fluidez lectora oral y pocas veces se realizan evaluaciones de FLS en L1 y en L2.

\subsection{Base teórica de la adquisición de la fluidez lectora en L1}

Un modelo que ha servido de base teórica para comprender las diferencias individuales en la adquisición de las destrezas lectoras en L1 es el de Visión Simple de la Lectura (Gough \& Tunmer, 1986; Hoover \& Gough, 1990). Este marco teórico explica la comprensión lectora como el resultado de la interacción entre el proceso de Decodificación (identificación de palabras escritas) y la Comprensión Lingüística (oral). Dentro de este modelo simple de la lectura, la fluidez lectora actúa como componente mediador que sirve de puente hacia la comprensión lectora (Pikulski \& Chard, 2005; Silverman, Speece, Harring, \& Ritchey, 2013). Desde este paradigma, Hiebert et al. (2012: 36) definen la fluidez lectora como "la habilidad de decodificar y comprender en una misma fijación ocular". Para un lector novel en L1 o un lector con dificultades de decodificación léxica, la unidad de reconocimiento visual de una palabra sería la letra (c-a-s-a) mientras que para un lector fluyente sería la palabra (casa), un indicativo de que los procesos previos para la identificación de palabras han sido automatizados. Este rasgo implica que la fluidez es una destreza relacionada con un constructo teórico crucial para su conceptualización, la automaticidad.

La base teórica cognitiva de la fluidez lectora son la Teoría de la Automaticidad de LaBerge \& Samuels (1974) y la Teoría de la Eficiencia Verbal (Perfetti, 1985). En ambas, las destrezas complejas como la fluidez lectora se explican atendiendo a que la lectura fluida requiere de la ejecución coordinada y eficaz de un conjunto de subdestrezas, a la vez que los recursos cognitivos de naturaleza atencional son limitados. Los seres humanos no solemos realizar más de un proceso a la vez, salvo que realicemos una tarea y luego otra, o que una tarea esté lo suficientemente bien aprendida y automatizada como para poder situar la atención en otra. En general, la teoría de la automaticidad y de la eficiencia verbal se basan en la reubicación de los procesos atencionales para la liberación de la memoria de trabajo. Es decir, un lector eficiente emplearía $100 \mathrm{~ms}$ en identificar la palabra casa dejando los otros $200 \mathrm{~ms}$ para comprender su significado en una lectura silenciosa (Samuels, Hiebert, \& Rasinski, 2014). Es decir, la fluidez lectora ha de ser entendida dentro del marco de la comprensión, porque la lectura rápida y correcta de palabras sin comprensión no sería fluidez sino velocidad (Rasinski \& Samuels, 2011).

En cuanto a la lectura silenciosa, antes se pensaba que la FLS era simplemente la manifestación subyacente de una misma habilidad lectora. Hoy se sabe que son constructos similares pero diferenciados. Van den Boer et al. (2014) comparó las destrezas que subyacen a la lectura oral y a la lectura silenciosa. Partiendo de tres indicadores (conciencia fonológica, 
velocidad de nombramiento y espacio de atención visual) como destrezas subyacentes, concluyó que la conciencia fonológica mostraba un comportamiento similar en ambos modos de lectura, si bien en la lectura silenciosa era menos notable su activación debido a que la forma ortográfica de las palabras podía activar directamente el significado. Por esta razón, el indicador de alcance visual resultó ser un índice importante en la lectura silenciosa, frente a la velocidad de nombramiento de palabras más vinculado con la fluidez lectora oral. Los resultados de la investigación de Zhao et al. (2016) confirman también estos hallazgos. Estos estudios, por tanto, indican que la atención visual podría ser interesante para diagnosticar problemas de lectura, así lo comprueba Gagliano et al. (2015) en el caso de la detección de la dislexia mediante la lectura fluida silenciosa.

\subsection{Base teórica de la adquisición de la fluidez lectora en $\mathbf{1 2}$}

La particularidad del proceso de adquisición de la habilidad lectora en segunda lengua recomienda mantener cierta precaución antes de extrapolar conclusiones de la L1 a la L2, porque plantea retos diferentes y añade a la compleja naturaleza del proceso lector la interacción e interferencia mutua de dos sistemas lingüísticos (Bernhardt, 2011; Fonseca-Mora \& Fernández-Corbacho, 2017; Koda, 2007a, 2007b). Los individuos adultos con una alfabetización previa nunca comienzan el desarrollo de su proceso lector desde cero, siempre saben algo. De acuerdo con la Teoría de la Interdependencia Lingüística de Cummins (1979), las habilidades lectoras en L1 pueden ser transferidas a la L2. De hecho, Bernhardt (2011) estipula que alrededor del $20 \%$ de la varianza en la comprensión lectora de jóvenes adultos se podría explicar por el nivel de literacidad en L1. Por otro lado, mientras que el lector adulto en L1 ya aprendió a leer en la escuela después de haber adquirido previamente un caudal lingüístico oral importante en su lengua materna durante la infancia, el lector adulto en L2 adquiere vocabulario oral a la vez que desarrolla su competencia lectora en L2. Esto podría explicar, por ejemplo, la mayor confianza en la decodificación ortográfica (visual) que en la decodificación fonológica (oral) de los lectores no nativos en los estadios iniciales, especialmente en los casos en que la relación entre grafema y fonema en la L2 es difusa (Oh, 2016). Hay un consenso general en reconocer el factor interlingüístico en el proceso de adquisición de la competencia lectora en L2. Así, la menor o mayor distancia lingüística entre la L1 y la L2 afectará al desarrollo de la fluidez lectora oral y silenciosa. La hipótesis de la ortografía opaca de Katz \& Frost (1992) sostiene que, cuando los individuos afrontan el aprendizaje de la lectura de lenguas (como el inglés), cuya relación entre grafemas y fonemas no es tan directa o transparente (como ocurre en español), su proceso de aprendizaje y la adquisición de habilidades como la fluidez lectora requieren de más tiempo.

En segundas lenguas, la investigación sobre la fluidez lectora silenciosa en adultos continúa siendo aún escasa en relación con la presencia de estudios en lengua materna. Lems (2003), en una de las primeras aproximaciones al estudio de la fluidez lectora oral y la lectura silenciosa, encontró que el nivel de conocimiento lingüístico de la L2 (gramática y vocabulario) correlacionaba de manera más significativa con la comprensión lectora mientras que la fluidez mantenía una relación significativa débil. Jeon (2012) también fracasa en su 
intento de conceptualizar el rol de la fluidez lectora en L2, el resultado de su investigación ofrece las mismas conclusiones que Lems (2003). El meta-análisis de Jeon y Yamashita (2014) refleja un panorama en el que los promedios de corrección gramatical en los estudios sobre comprensión de textos escritos en L2 correlacionan con una magnitud más fuerte que la fluidez.

Según Grabe (2010), una de las dificultades del estudio de la fluidez lectora en L2 guarda relación con un conflicto que parece estar marcadamente presente en el ámbito de la investigación en adquisición de competencia lectora en lengua extranjera: el debate entre corrección (gramática) o fluidez. En el ámbito de la L2, parece que aún sigue pendiente la respuesta a la pregunta de Alderson (1984), "Reading in a foreign language: A Reading problema or a language problem?" Jeon y Yamashita (2014) respondieron que se trata de un problema lingüístico, no cognitivo, pero obviaron aspectos como la velocidad de procesamiento (Oh, 2016), siendo ésta una habilidad cognitiva fundamental que afecta al proceso lector.

Este estado de la cuestión refleja, pues, la necesidad de estudiar la FLS atendiendo a las peculiaridades del lector de L2 de acuerdo con su nivel de competencia comunicativa en la segunda lengua y observando no solo su conocimiento del componente lingüístico de la lengua meta sino también sus habilidades lectoras en la lengua materna.

\section{MÉtodo}

\subsection{Participantes}

La muestra objeto de estudio estuvo conformada por 47 alumnos españoles de la asignatura Didáctica de la lengua extranjera, del $2^{\circ}$ curso del grado de Educación Infantil impartida en régimen de inmersión total en inglés. En cuanto a la formación lingüística previa, el $10 \%$ había cursado únicamente inglés en contexto escolar, mientras que el $90 \%$ había recibido clases de inglés y francés durante una media de 10 años. Al no haber cursado inglés desde Bachillerato y al ser la única asignatura que cursan en inglés en la carrera, en general, el alumnado se mostró temeroso de ser capaz de comprender e interactuar en la L2.

\subsection{Hipótesis}

Ante la ausencia de resultados definitivos sobre la trayectoria de la FLS en diferentes niveles de comprensión lectora en L2, en este estudio se compara el comportamiento de la lectura silenciosa en L1 y en L2 del alumno AICLE, tomando como indicador predictivo de FLS la capacidad de identificar con eficiencia palabras conectadas en un texto. Al estudiar el comportamiento de la lectura en silencio de un texto en L1 (español) y otro de similares características en la L2 (inglés), nos planteamos las siguientes hipótesis:

- Existe una relación significativa entre la FLS del alumno en L1 y su FLS en L2.

- Existe una relación significativa entre la fluidez en L2 con el nivel de comprensión lectora del alumno en L2.

- Se observan diferencias significativas entre los grupos conformados por el nivel de comprensión lectora según Dialang (A1, A2, etc.) en las puntuaciones de FLS en L2. 
- Se observan diferencias significativas en los niveles de comprensión en la puntuación en fluidez lectora en L2.

\subsection{Instrumentos y procedimiento de recogida de datos}

La recogida de datos se realizó ad hoc mediante dos pruebas: el test de diagnóstico de nivel de comprensión lectora en L2 (DIALANG) y los tests de lectura fluida silenciosa en español y en inglés. Dialang es una prueba de diagnóstico on line, creada a partir de los descriptores generales del Marco Común Europeo de Referencia (MCER) (Consejo de Europa, 2002), que permite clasificar el nivel de comprensión lectora de los usuarios desde un A1 (Etapa Usuario Básico) a un C2 (Etapa usuario competente). El test de comprensión lectora se desarrolló en tres partes. En la primera, el usuario recibió una prueba de reconocimiento léxico. En función de su resultado, el programa informático selecciona los ítems de lectura más adecuados para poder diagnosticar su nivel. Esta prueba consta de tres columnas de 25 palabras y pseudo-palabras en L2, en total, 75 elementos. El usuario decide en cada uno de ellos si es o no una palabra en L2. A continuación, la segunda parte era un cuestionario para conocer la percepción del alumno acerca de su habilidad de comprender un texto escrito en L2. Esta prueba determinó el nivel de la prueba que se proporciona al alumno. Por último, el alumno accedió a un conjunto de pruebas de comprensión, muy breves, basadas en textos de diferente naturaleza (instruccionales, informativos, argumentativos, etc.) con una tipología dinámica y variada de actividades (selección múltiple, escribir un ítem de respuesta abierta, etc.).

Los tests de lectura fluida silenciosa para el reconocimiento de palabras en L1 y en L2 son pruebas de creación propia, en un formato muy similar a otros tipos de test que han sido empleados también para conocer la naturaleza de la FLS, como es el caso del TOSCRF (Hammill, Wiederholt, \& Allen, 2006). Éste es un test administrado en grupo y que se mide con referencia a una norma, aunque en L2 no se dispone de un marco normativo para discernir niveles de FLS que permita conocer su comportamiento y desarrollo a lo largo del proceso de aprendizaje. Esta prueba mide la rapidez con la que los estudiantes pueden identificar palabras individuales dentro de un texto. Las palabras impresas aparecen sin espacios ni signos de puntuación. A diferencia del TOSCRF, las letras aparecen en minúscula siguiendo el precepto de Hiebert \& Reutzel (2014: 37) de que "las palabras minúsculas ofrecen al ojo lector un Skyline de las palabras". Los estudiantes tuvieron tres minutos para reconocer tantas palabras como les fuera posible utilizando barras de separación con el bolígrafo. El resultado total fue derivado del número de palabras correctamente identificadas en el espacio de tiempo señalado. Según Hammill et al. (2006), la validez estimada de esta prueba varía de 0.67 a 0.85 en relación con otras medidas de lectura validadas.

Esta prueba fue suministrada primero en la L2 (inglés) y después en la L1 (español) con la intención de que no hubiera un efecto de anticipación. El contenido del texto en L2 y L1 correspondía a extractos de la Declaración de los Derechos Humanos, cuya elección estuvo motivada por su nivel de legibilidad (texto expositivo) y por su independencia cultural. 


\subsection{Análisis de datos}

En primer lugar se llevó a cabo un análisis de normalidad de las variables cuantitativas, observando que no seguían una distribución normal, por lo que se optó por pruebas no paramétricas (correlación de Spearman, y contrastes de Kruskal Wallis y U de Mann - Whitney) para el estudio de las relaciones entre estas variables y las diferencias entre grupos. En estos análisis se produjo una pérdida de 5 sujetos, que no llevaron a cabo las pruebas de nivel de L2, por lo que las pruebas de contraste se realizaron entre 47 sujetos. Se incluyen análisis descriptivos y de frecuencias de las variables categóricas objeto de estudio. Los datos se analizaron mediante el paquete estadístico SPSS en su versión 23.

\section{Resultados}

La tabla 1 contiene los principales descriptivos de las variables objeto de estudio. La mayoría del alumnado posee un nivel de usuario básico de comprensión, siendo el nivel más frecuente el A1 con 25 estudiantes (48.1\%).

Tabla 1. Estadísticos descriptivos

\begin{tabular}{|c|c|c|c|c|c|c|c|c|}
\cline { 2 - 9 } \multicolumn{1}{c|}{} & $\mathrm{N}$ & Mín & Max & Media & Sd & K-S & gl & $\mathrm{p}$ \\
\hline Dialang & 51 & 180 & 769 & 403,88 & 156,331 &, 130 & 40 &, 086 \\
\hline FLSL1 & 52 & 68 & 211 & 131,288 & 28,935 &, 076 & 40 &, $200^{*}$ \\
\hline FLSL2 & 47 & 19 & 108 & 55,234 & 21,539 &, 077 & 40 &, $200^{*}$ \\
\hline $\begin{array}{c}\text { Reading Test } \\
\text { Level } \\
(\mathrm{n}=47)\end{array}$ & $\mathrm{N}$ & $\%$ Válido & & & & & \\
\hline A1 & 25 & 48.1 & & & & & & \\
\hline A2 & 17 & 32.7 & & & & & \\
\hline B-C & 10 & 19.2 & & & & & \\
\hline Total & 52 & 100 & & & & & \\
\hline * Esto es un límite inferior de la significación verdadera. \\
Corrección de significación de Lilliefors \\
\hline
\end{tabular}

En cuanto a las hipótesis correlacionales, se ha llevado a cabo una Correlación no paramétrica de Spearman, observándose la existencia de una correlación significativa positiva, entre la fluidez lectora silenciosa del alumnado en ambas lenguas $\left(r_{\mathrm{s}}=.390, \mathrm{p}=.003\right)$ con los resultados del test de lectura DIALANG $\left(\mathrm{r}_{\mathrm{s}}=.422, \mathrm{p}=.003\right)$.

Dada la relación significativa entre estas variables, se puso a prueba la existencia de diferencias significativas entre grupos coherentes con los descriptores del MCER (2002), con el fin de observar el desarrollo de la trayectoria de la FLS por niveles de comprensión 
lectora. Para ello, se agruparon los resultados de la Etapa Nivel Independiente (B1 y B2) con la Etapa Nivel Competente (Niveles $\mathrm{C} 1$ y C2), obteniéndose tres grupos diferenciados (A1, A2, B-C). En la tabla 2 se puede observar que existen diferencias significativas entre niveles $(\mathrm{KW}=8.467, \mathrm{p}=.015)$, más concretamente entre los niveles $\mathrm{A} 1-\mathrm{BC}\left(\mathrm{U}_{\mathrm{MW}}=\right.$ $37.5, \mathrm{p}=.004)$, no existiendo diferencias entre los niveles A1 - A2 y entre los niveles A2 - BC. El tamaño del efecto observado en los contrastes entre grupos oscila entre mediano (A1 - A2) y elevado (A1 - BC).

Tabla 2. Pruebas de contraste entre grupos

\begin{tabular}{|c|c|c|c|c|c|}
\hline & RTL & $\mathrm{N}$ & $\begin{array}{l}\text { Rango } \\
\text { promedio }\end{array}$ & & \\
\hline \multirow[t]{5}{*}{ Kruskal Wallis (L2) } & A1 & 21 & 18,52 & $\chi_{(\mathrm{a})}^{2}$ & 8,467 \\
\hline & A2 & 16 & 25,13 & $\mathrm{gl}$ & 2 \\
\hline & $\mathrm{B}-\mathrm{C}$ & 10 & 33,7 & $\mathrm{p}$ & $0,015^{*}$ \\
\hline & Total & 47 & & & \\
\hline & $\mathrm{A} 1-\mathrm{A} 2$ & $\mathrm{~A} 1-\mathrm{BC}$ & $\mathrm{A} 2-\mathrm{BC}$ & & \\
\hline U de Mann-Whitney ${ }^{\mathrm{b}}$ & 121 & 37,5 & 50,5 & & \\
\hline $\mathrm{Z}$ & $-1,5$ & $-2,854$ & $-1,556$ & & \\
\hline Sig. asintótica (bilateral) & 0,15 & $0,004 *$ & 0,12 & & \\
\hline Cohen $\mathrm{d}$ & .533 & 1.311 & .798 & & \\
\hline EffectSize (r) & .257 & .548 & .371 & & \\
\hline \multicolumn{6}{|c|}{ a. Variable de agrupación: Reading Test Level } \\
\hline * Se observan diferencias & rupos & & & & \\
\hline
\end{tabular}

Con el fin de observar la evolución de la FLS, en la tabla 3 se incluyen los estadísticos descriptivos por grupos de nivel de comprensión lectora, observándose que las puntuaciones van ascendiendo según se asciende en el nivel de lectura.

Tabla 3. Estadísticos descriptivos por niveles de comprensión lectora

\begin{tabular}{|c|c|c|c|c|c|c|c|}
\hline & & & Media & Mínimo & Máximo & $\mathrm{Sd}$ & Mediana \\
\hline \multirow[t]{3}{*}{ RTL } & $\mathrm{A} 1$ & $\mathrm{~L} 2$ & 46,19 & 19,00 & 78,00 & 17,41 & 44,00 \\
\hline & $\mathrm{A} 2$ & L2 & 56,00 & 26,00 & 91,00 & 19,34 & 56,00 \\
\hline & $\mathrm{B}-\mathrm{C}$ & L2 & 73,00 & 47,00 & 108,00 & 23,07 & 67,50 \\
\hline
\end{tabular}




\section{Discusión}

El objetivo principal de esta investigación era determinar si la medición de la FLS en L1 y en L2 permitía conocer la transferencia de habilidades lectoras de L1 a L2 o la trayectoria de la FLS a lo largo de los niveles de competencia lectora, para poder identificar así las posibles dificultades de los estudiantes universitarios en cada fase del proceso dentro de un contexto AICLE.

En nuestra hipótesis inicial nos planteábamos si independientemente del nivel de comprensión lectora del alumno en L2 podría observarse una transferencia de habilidades en FLS de L1 a L2. Los resultados de los tests indican que hay una relación significativa entre ambas variables, aunque la magnitud de tal correlación es débil $\left(\mathrm{r}_{\mathrm{s}}=.390, \mathrm{p}=.003\right.$. En la literatura especializada en adquisición del proceso lector en L2, se reconoce ampliamente la importancia de las habilidades lectoras en L1 como factor explicativo y predictivo del buen desarrollo de la competencia lectora en L2 (Ziegler \& Goswami, 2006; Koda, 2007a;Bernhardt, 2011; Fonseca-Mora \& Fernández-Corbacho, 2017). La novedad de nuestro estudio ha sido analizar la posible transferencia en la lectura silenciosa, observando su correlación en L1 y en L2, para tratar de entender el comportamiento del modo de lectura más habitual en individuos adultos y partiendo del supuesto de que la lectura silenciosa implica procesos similares a los de la lectura oral, pero también la existencia de comportamientos subyacentes diferentes (Hiebert et al., 2012; van den Boer et al., 2014; Zhao et al., 2016). En este estudio, la FLS fue definida como la capacidad de decodificar palabras (corrección) conectadas en un texto sin espacios, dentro de un tiempo limitado de tres minutos (velocidad), y aislándola de la comprensión lectora, en cierta medida. Así, la observación de un componente predictivo de fluidez, como es el caso de la decodificación léxica (Fuchs, Fuchs, Hosp, \& Jenkins, 2001) nos ha permitido analizar la capacidad de los lectores de reconocer los límites ortográficos del léxico presente en el texto desde el nivel A1. El análisis de estos datos nos muestra que hay individuos con buena fluidez lectora en L1 que superan en número de palabras identificadas en L2 a otros individuos de mayor nivel de competencia lectora (B1 y B2) pero que tienen menos habilidad de reconocer palabras en L1. Esto significa que el alumno A1 puede llegar a decodificar visualmente más palabras que un B1 o un B2, pero no implica que comprenda más texto en L2. Estas evidencias se relacionan con estudios sobre la relación de la fluidez lectora oral y la comprensión en estudiantes de L2, cuyos resultados mostraban que el conocimiento lingüístico de la L2 correlaciona más fuerte que la fluidez lectora con la comprensión escrita (Jeon, 2012; Jeon \& Yamashita, 2014; K. Lems, 2003; Lems, 2012a, 2012b). Estos alumnos tenían promedios muy altos de corrección gramatical (Lems, 2003; Jeon, 2012) pero unos promedios de fluidez muy bajos. Esto parece evidenciar que un mayor conocimiento de gramática en L2, junto con otros componentes importantes como el vocabulario (Oh, 2016) conducen a una mayor comprensión, pero no tiene por qué conducir a una mayor fluidez lectora. Así, para igualar la tasa de habilidad de reconocimiento de palabras entre la L1 y la L2, lo cual contribuiría a mejorar la fluidez, es probable que el alumno necesite aumentar su conocimiento de la lengua extranjera pero también es probable que necesite una mayor experiencia con la lectura en L2 para aumentar su alcance de reconocimiento visual. 
No es de extrañar que un lector de L2 universitario sea capaz de transferir su habilidad de decodificación visual-ortográfica en L1 y tener así un alcance de reconocimiento visual similar al nivel de promedio obtenido por otro alumno de un nivel de competencia más alto en el test de fluidez lectora, ya que la distancia entre dos lenguas alfabéticas en la lectura silenciosa es menor que en la oralidad (español-inglés) y menor también que la distancia visual existente entre una lengua alfabética y otra logográfica (español-chino). En el ámbito de la adquisición de la lectura en L2, hay también un consenso a la hora de reconocer el impacto de la naturaleza del sistema ortográfico en el desarrollo de la fluidez lectora oral en L1 y en L2. De acuerdo con la hipótesis de la ortografía opaca (Katz \& Frost, 1992), el proceso de la lectura es diferente cuando los sistemas ortográficos son opacos, es decir, cuando la relación entre grafía (grafema) y sonido (fonema) es difusa, como es el caso del inglés (Share, 2008).

Lo menos predecible en nuestro estudio han sido los niveles de dominio del inglés tan bajos detectados en el nivel terciario de nuestro sistema educativo. Por ello, la implementación de una metodología AICLE con especial atención a la lectura de textos nos parece altamente recomendable. Teniendo en cuenta que en clase disponemos de diversidad de niveles de competencia en L2, la lectura de textos en L2 puede ser incluso un primer paso que sirva de apoyo a la destreza de la comprensión oral. De acuerdo con los resultados de Oh (2016), el desarrollo de la competencia lectora en L2 parece ir en una dirección inversa al desarrollo de la competencia lectora en L1. En una situación de segundas lenguas, el lector de L2 no dispone del caudal de léxico oral ni del nivel de competencia lingüística que posee el lector de L1 cuando accede a aprender a leer. Por ello, aumentar su experiencia lectora con textos en L2 podría facilitar tanto su comprensión oral como su comprensión escrita.

La segunda de nuestras hipótesis se dirigía a conocer si los comportamientos en el test de FLS en L2 podrían mantener algún tipo de relación con los resultados del test de diagnóstico. Los datos de nuestro estudio nos permiten observar que hay una relación significativa entre fluidez y comprensión, pero la magnitud de la correlación es débil $\left(\mathrm{r}_{\mathrm{s}}=.422, \mathrm{p}\right.$ $=.003$ ). Como hemos señalado anteriormente, en L1, el estudio del desarrollo de la lectura en inglés con niños reconoce la importancia de la memoria fonológica en primer lugar y de la importancia secundaria de la memoria visual u ortográfica, así, al menos, se plantea en la hipótesis de la autoenseñanza de Share (2008). En L2, sin embargo, tal vez esto suceda al contrario. La menor exposición del lector de L2 a la oralidad, hace que se inviertan los procesos y que el lector acceda al significado desde la decodificación ortográfica (Lems, 2003; Oh, 2016). Esto explicaría la relación débil de la correlación entre el reconocimiento eficiente de palabras en L2 y el puntaje en la prueba de comprensión lectora de acuerdo con los descriptores del MCER (2002). Esta circunstancia implicaría la posición de ventaja a nivel de comprensión que se produce en la lectura silenciosa en adultos ya alfabetizados en una L1 (Lems, 2003). Al ser una lectura que exige una mayor concentración visual, el hecho de ser lector competente en una L1 puede favorecer el reconocimiento de palabras (memoria visual) en la lectura silenciosa. En nuestro estudio los estudiantes ya habían recibido instrucción y poseen, por tanto, un conocimiento lingüístico de L2 y de vocabulario. Es posible que esta destreza haya permitido acceder directamente al significado a aquellos lectores de niveles más elementales. Obviamente, las condiciones en el aprendizaje de la 
lectura en L1 son diferentes. En ese escenario los niños necesitan traducir la letra a sonidos para acceder a su lexicón mental y este procedimiento, además, demuestra ser más efectivo en el desarrollo de la habilidad lectora en L2. Así, un contexto AICLE puede crear un entorno inmersivo y proveer ese caudal sonoro que impulse el desarrollo de la competencia lectora del alumno. El componente gramatical y léxico es un fuerte predictor de comprensión lectora en L2, la habilidad lectora en L1 también contribuye en algo al desarrollo de esa competencia, pero la experiencia oral es tan fundamental como la experiencia lectora en L2 (Lems, 2017). Los lectores de L2 pueden transferir su capacidad de decodificar palabras en modo visual y saber leer algo, pero serán mejores lectores cuando desarrollen también su conciencia fonológica. Especialmente, si la lengua posee un sistema ortográfico opaco como el inglés (Fonseca-Mora y Fernández-Corbacho, 2017).

En la tercera hipótesis de nuestro estudio nos planteamos si es posible analizar la trayectoria del desarrollo de la FLS a lo largo de los diferentes niveles de comprensión lectora en L2. Los resultados no permiten observar una relación significativa de crecimiento entre la FLS y los niveles de comprensión en L2. El tamaño de nuestra muestra es reducido y desigual. Hay una concentración de alumnos en el nivel Usuario Básico (A1 y A2) con 37 representantes, un escenario más pobre en Usuario Independiente (B1 y B2) con solo 9 y una escasez enorme de Usuarios Competentes ( $\mathrm{C} 1$ y $\mathrm{C} 2$ ) con solo 1 representante. Como decíamos al principio, los resultados del Test de Diagnóstico (DIALANG) son sorprendentes, pues no esperábamos una presencia tan numerosa de alumnos en los niveles A1 y A2. No obstante, en la Tabla 3 se puede observar un eje de crecimiento de la FLS intergrupal: A1- A2 (46, 19 - 50), B-C (73).

Nuestro test de FLS es una prueba que permite observar la eficiencia del alumno en la decodificación de palabras, pero no mide la soltura de la velocidad lectora con comprensión. Como señala Hiebert (2014), la clave parece estar en la velocidad dentro de los límites que permite la comprensión y una adecuada conciencia fonológica en L2. No hay muchas actividades de aceleración de la lectura. Taguchi, Takayasu-Maass, \& Gorsuch (2004) comprueban la validez de una técnica cuya eficacia ha sido contrastada en el ámbito de la fluidez lectora oral en L1, la lectura repetida (Repeated Reading), en el contexto de inglés L2. Esta prueba sería difícil transferirla al contexto AICLE universitario, pero el aumento de la experiencia lectora y la naturaleza casi inmersiva que proporciona este modelo podrían ser suficientes para mejorar la velocidad de la lectura en L2.

Nuestra última hipótesis se centra en conocer si es posible observar diferencias de comportamiento intergrupales de la FLS. Los resultados indican que hay una diferencia significativa entre los grupos A1 y B-C. Se observa una relación significativa moderada $\left(\mathrm{U}_{\mathrm{MW}}=37.5, \mathrm{p}=.004\right)$ entre dos grupos que se encuentran en dos etapas distintas (Grupo A1- Grupo BC) pero el bajo número de participantes con niveles B1 o superior no permite aún determinar más diferencias. El análisis de los resultados en las Tablas 2 y 3 muestra un comportamiento intergrupal de la FLS bastante lento. Los resultados del Test de FLS en L1 y en L2 permitieron observar que en L2 la eficiencia en la segmentación de palabras disminuía notablemente en comparación con la eficiencia demostrada en L1 en la gran mayoría de los casos. 


\section{Consideraciones finales}

El objetivo central de este estudio es observar las diferencias individuales en el logro de la comprensión escrita a partir de la observación de la FLS en L1 y en L2. En L1, un modelo teórico para conocer el comportamiento de las diferencias individuales es el de la Visión Simple de la Lectura(Gough \& Tunmer, 1986; Hoover \& Gough, 1990). Pero en una situación de L2, este modelo tiende a desestabilizarse. Una de las claves, como señala Share (2008), es el nivel de automaticidad al que ha llegado el proceso de recodificación fonológica en L1. La fluidez es un constructo que encuentra su base teórica dentro de la teoría cognitiva de la automaticidad desde los niveles inferiores de procesamiento (unidades más pequeñas como las letras, los sonidos, las palabras). En una situación de lectura silenciosa en inglés L2, puede ocurrir que en lugar de activarse en primer lugar el proceso de decodificación fonológica, también presente en la lectura silenciosa (van den Boer et al., 2014), el alumno active antes una estrategia de lectura de orden mayor (el reconocimiento ortográfico y semántico) para vincular palabra y significado en ausencia de una representación mental fonológica de la L2 correcta, al menos en los niveles iniciales del desarrollo de la competencia lectora en L2. En este sentido, el rol de la fluidez lectora como factor mediador de la comprensión de textos escritos es posible que se vea alterado en el contexto de la L2. Nuestro estudio es una aproximación al complejo fenómeno de la FLS en la lectura de L2 de adultos. En futuras investigaciones sobre la FLS es imprescindible también analizar el procesamiento fonológico y la velocidad de procesamiento.

\section{Agradecimientos}

Este estudio ha sido financiado por el Ministerio de de Economía y Competitividad de España a través del proyecto $\mathrm{I}+\mathrm{D}+\mathrm{i}$ «Aptitud musical, fluidez lectora y percepción intercultural de estudiantes universitarios europeos» Referencia: FFI2016-75452-R 1

\section{REFERENCIAS}

Alderson, J.C. (1984). Reading in a foreign language: A reading problem or a language problem? In J.C. Alderson \& A.H. Urquhart (Eds.), Reading in a foreign language (pp. 1-27). New York: Longman.

Bernhardt, E.B. (2011). Understanding advanced second-language reading. New York, NY: Routledge. Ciuffo, M., Myers, J., Ingrassia, M., Milanese, A., Venuti, M., Alquino, A., Baradello, A., Stella, G., y Gagliano, A. (2017). How fast can we read in the mind? Developmental trajectories of silent reading fluency. Reading and Writing, 30(8), 1667-1686.

Consejo de Europa. (2002). Marco Común Europeo de Referencia para las Lenguas.Strasburgo.

Cummins, J. (1979). Linguistic interdependence and the educational development of bilingual children. Review of Educational Research, 49(2), 222-251.

Eurydice. (2011). La enseñanza de la lectura en Europa: contextos, políticas y prácticas. (Ministerio de Educacion, Ed.). Bruselas: Agencia Ejecutiva en el ámbito Educativo Audiovisual y Cultural. 
Fonseca-Mora, M.C. \& Fernández-Corbacho, A. (2017). Procesamiento fonológico y aprendizaje de la lectura en lengua extranjera. Revista Española de Lingüistica Aplicada/Spanish Journal of Applied Linguistics, 30(1), 166-187.

Fuchs, L.S., Fuchs, D., Hosp, M.K. \& Jenkins, J.R. (2001). Oral reading fluency as an indicator of reading competence: A theoretical, empirical, and historical analysis. Scientific Studies of Reading, 5(3), 239-256.

Gagliano, A., Ciuffo, M., Ingrassia, M., Ghidoni, E., Angelini, D., Benedetto, L.,y Stella, G. (2015). Silent reading fluency: Implications for the assessment of adults with developmental dyslexia. Journal of Clinical and Experimental Neuropsychology, 37(9), 972-80.

Gough, P.B., \& Tunmer, W.E. (1986). Decoding, reading, and reading disability. Remedial and Special Education, 7(1), 6-10.

Grabe, W. (2010). Fluency in reading - Thirty-five years later. Readning in a Foreign Language, 22(1), 71-83.

Hammill, D.D., Wiederholt, J.L., \& Allen, E.A. (2006). Test of silent contextual reading fluency. Austin, TX: PRO-ED.

Hiebert, E.H., Samuels, S.J., \& Rasinski, T. (2012). Comprehension-based silent reading rates: What do we know? What do we need to know? Literacy Research and Instruction, 51(2), $110-124$.

Hiebert, E. \& Reutzel, D.R. (2014). Revisiting silent reading: New directions for teachers and researchers. .: Santa Cruz, CA : I. TextProject, Ed.

Hoover, W.A., \& Gough, P.B. (1990). The simple view of reading. Reading and Writing, 2(2), $127-160$.

Jeon, E.H. (2012). Oral reading fluency in second language reading. Reading in a Foreign Language, 24(2), 186-208.

Jeon, E.H., \& Yamashita, J. (2014). L2 Reading comprehension and its correlates: A meta-analysis. Language Learning, 64(1), 160-212.

Katz, M., \& Frost, R.(1992). Orthography, phonology, morphology and meaning. In R. Frost \& L. Katz (Eds.), Advances in Psychology Vol. 94. Amsterdam: Elsevier Science.

Koda, K. (2007a). Insights Into Second Language Reading: A Cross-Linguistic Approach.Reading in a Foreign Language (Vol. 18). New York, NY: Cambridge University Press.

Koda, K. (2007b). Reading and Language Learning: Crosslinguistic constraints on second language reading development. Language Learning, 57, 1-44.

Kuhn, M.R., Schwanenflugel, P.J., Meisinger, E.B., Levy, B.A., \& Rasinski, T.V. (2010). Aligning theory and assessment of reading fluency: Automaticity, prosody, and definitions of fluency. Reading Research Quarterly, 45(2), 230-251.

LaBerge, D. \& Samuels, S.J. (1974). Toward a theory of automatic information processing in reading. Cognitive Psychology, 6(2), 293-323.

Lems, K. (2003). Adult ESL oral reading fluency and silent reading comprehension. NationalLouis University.

Lems, K. (2012a). Reading fluency and comprehension in adult English language learners. In T.V. Rasinski, C. Blachowicz \& K. Lems (Eds.), Fluency instruction: Research based best practices (2nd ed., pp. 243-254). New York: The Guilford Press.

Lems, K. (2012b). The effect of L1 orthography on the oral reading of adult English language learners. Writing Systems Research, 4(1), 61-71. 
Lems, K. (2017). Talkin oracy and SVR. Illinois Reading Council Journal, 45(4), 74-78.

National Reading Panel. (2000). Teaching children to read: An evidence-based assessment of thescientific literature on reading and its implications for reading instruction: Reports of the sub-groups. Bethesda, MD.

Oh, E. (2016). Comparative studies on the roles of linguistic knowledge and sentence processing speed in L2 listening and reading comprehension in an EFL tertiary setting. Reading Psychology, 37(2), 257-285.

Perfetti, C.A. (1985). Reading ability. New York: Oxford University Press.

Pikulski, J.J. \& Chard, D.J. (2005). Fluency: Bridge between decoding and reading comprehension. The Reading Teacher, 58(6), 510-519.

RAND (2002). Reading for understanding: Toward an $R \& D$ program in reading comprehension. Santa Monica, CA: Office of Educational Research and Improvement.

Rasinski, T. (2014). Fluency matters. International Electronic Journal of Elementary Education, $7(1), 82-91$.

Rasinski, T. \& Samuels, S.J. (2011). Reading fluency: What it is and what it is not. In S. J. Samuels \& A. E. Farstrup (Eds.), What research has to say about reading instruction (pp. 94-114). Newark, DE: International Reading Association.

Samuels, S.J., Hiebert, E.H., \& Rasinski, T.V. (2014). Eye movements make reading possible. In E. H. Hiebert \& D. R. Reutzel (Eds.), Revisiting silent reading: New directions for teachers and researchers (pp. 24-44). Santa Cruz, CA: TextProject, Inc.

Share, D.L. (2008). On the anglocentricities of current reading research and practice: The perils of overreliance on an "outlier" orthography. Psychological Bulletin, 134(4), 584-615.

Silverman, R.D., Speece, D.L., Harring, J.R., \& Ritchey, K.D. (2013). Fluency has a role in the simple view of reading. Scientific Studies of Reading, 17(2), 108-133.

Taguchi, E., Takayasu-Maass, M., \& Gorsuch, G.J. (2004). Developing reading fluency in EFL : How assisted repeated reading and extensive reading affect fluency development. Reading in a Foreign Language, 16(2), 70-96.

Van den Boer, M., van Bergen, E., \& de Jong, P.F. (2014). Underlying skills of oral and silent reading. Journal of Experimental Child Psychology, 128, 138-151.

Zhao, J., Kwok, R.K.W., Liu, M., Liu, H., \& Huang, C. (2016). Underlying skills of Oral and silent reading fluency in Chinese: Perspective of visual rapid processing. Frontiers in Psychology, 7, 2082.

Ziegler, J.C. \& Goswami, U. (2006). Becoming literate in different languages: similar problems, different solutions. Developmental Science, 9(5), 429-436. 